\section{Cooling of Permanent Magnet Alloys in a Constant} Magnetic Field

THE investigations of Bozorth and Dillinger ${ }^{1}$ show that very striking increases in maximum permeability may be obtained by applying a magnetic field to high permeability nickel-iron alloys during cooling. So far as we are aware, corresponding investigations have not been made on materials of high coercivity.

We have recently carried out experiments with positive results on permanent magnet alloys of the nickel-aluminium-iron Mishima type by cooling in a

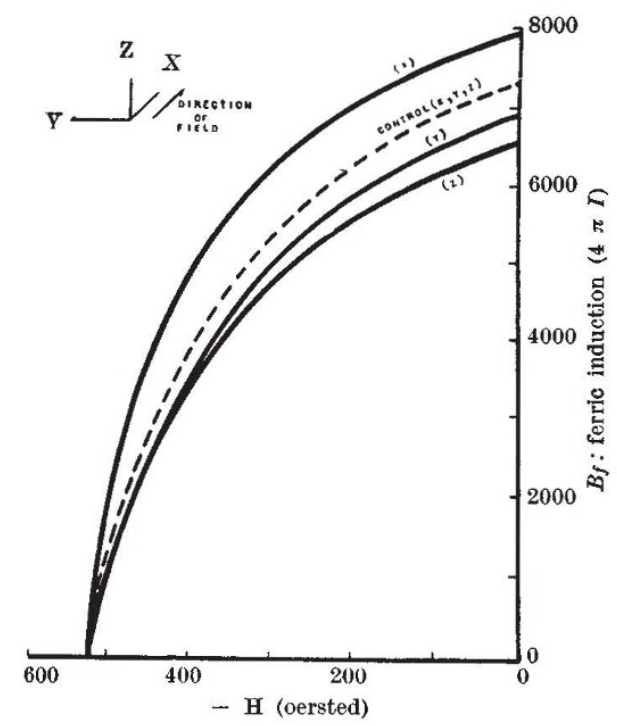

unidirectional magnetic field from $1,200^{\circ} \mathrm{C}$., and then measuring the demagnetization curves at room temperature. In general, we find the coercivity is not greatly affected, but that the remanence and the value of $B H_{\max }$. are increased. In the two directions at right angles, the results are practically identical with lowered values of remanence and $B H_{\max }$. Typical results for Alnico (nickel 18, aluminium 10, iron 54, cobalt 12, copper 6 per cent) are given in the accompanying graph, which refers to some experimental cubes of $4 \cdot 0 \mathrm{~cm}$. side. Care was taken to ensure that so far as possible similar thermal conditions were observed in making the control experiment.

In the direction of the field, the true $B H_{\max }$. value increased from $1.5 \times 10^{6}$ to $1.8 \times 10^{6}$, or 20 per cent. At right angles to the field direction it dropped to $1.35 \times 16^{6}$, or 10 per cent. The field strength $H$ was 4,400 oersted. If $I_{r}$ represents the remanent intensity of magnetization, and $I_{o}$ the saturation intensity, at room temperature, then we have found the ratio $I_{r} / I_{0}$ to be 0.72 in the direction of the constant applied magnetic field, 0.61 transverse to the direction of the field, and 0.67 for the control with no applied field during cooling.

An interpretation of these effects can be offered on lines similar to that put forward by Bozorth and Dillinger in connexion with their results. On cooling a ferromagnetic below the Curie point and through the temperature range in which plastic flow occurs, the magnetostrictive strains are to some extent relieved, and the actual direction of magnetization of each domain becomes an energetically favoured direction of magnetization. These directions would normally be at random through the material as a whole; but if a field is applied during cooling, they will be so distributed as to favour subsequent bulk magnetization parallel or antiparallel to the direction of the original applied field. In the limit, the normal six or eight equivalent easy directions for each domain (for cubic structures) would be replaced by two, and (for a particular direction) the remanent magnetization would then be equal to the saturation magnetization.

This state may be nearly approached with high permeability material. With permanent magnet alloys, for reasons which are qualitatively clear, the effect is relatively small, but it is large enough to be of possible technological value, and the determination of its magnitude may contribute to a more quantita. tive theoretical treatment of the properties of these alloys in relation to their structure ${ }^{2}$.

We wish to thank Prof. W. L. Bragg for his encouragement and for suggesting following up this line of investigation, and Dr. E. C. Stoner for helpful comments.

Research Department,

D. A. OrIver.

J. W. Shenden.

William Jessop and Sons, Ltd., Sheffield. June 28.

${ }^{1}$ Bozorth, R. M., and Dillinger, J. F., Physics, 6, 279 and 285 (1935). ${ }^{2}$ Bradley, A. J., and Taylor, A., Magnetism, p. 89 (Institute of Physics, 1938).

\section{Electrical Impedance of Nerve During Activity}

Alternating current impedance measurements made over a wide frequency range show that the membranes of many living cells, including those of several nerve fibres ${ }^{1}$, have electrical capacities of about one microfarad per square centimetre. The leakage conductance of the membranes of resting cells is small and there is considerable variation in the phase angle, which is assumed to be a measure of the dielectric loss. These same characteristics have been found from transverse impedance measurements, made with the current flow perpendicular to the cell axis, on the long single cell of the plant Nitella ${ }^{2}$ and the giant nerve fibre of the stellar nerve of the squid, Loligo pealii ${ }^{3}$.

Because of their large diameters, it has been possible to observe and measure, in both these cells, the change in transverse impedance during the passage of the wave of excitation, which is the nerve impulse in nerve and its analogue in Nitella. In Nitella $^{4}$, the membrane resistance falls from $10^{4}$ ohm cm. ${ }^{2}$ or more to a minimum of about $500 \mathrm{ohm}$ $\mathrm{cm} .^{2}$, while the membrane capacity decreases fifteen per cent with its phase angle unchanged. In the squid nerve ${ }^{5}$, the membrane resistance falls to about $100 \mathrm{ohm} \mathrm{cm.}{ }^{2}$ and the capacity change is about a one per cent decrease without alteration of the phase angle.

The time course of the impedance change is very similar in the two cells, although the Nitella is a thousand times slower. For the squid fibre measurements, the amplified output of a Wheatstone bridge was applied to a cathode ray oscillograph which gave a narrow horizontal trace when the bridge was balanced and a sweep circuit moved the spot across the screen. In the accompanying illustration, the nerve fibre was stimulated at one end and the band 